\title{
Is biliary lithiasis associated with pancreatographic changes?
}

\author{
M Barthet, C Affriat, J-P Bernard, P Berthezene, J-C Dagorn, J Sahel
}

\begin{abstract}
The aetiological role of biliary lithiasis for chronic pancreatitis remains controversial. Previous studies based on pancreatographic studies reported changes in the pancreatic duct system caused by biliary lithiasis. This study analysed retrospectively the endoscopic retrograde cholangiopancreatography of 165 patients presenting with biliary lithiasis and of 53 controls. Among the 165 patients, 113 had choledochal stones ( 53 with gall bladder stones, 50 had had a cholecystectomy, 10 with a normal gall bladder), 35 had gall bladder stones without choledochal stones, 17 had cholecystectomy for gall bladder stones. Pancreatograms were analysed by measuring the diameter of the pancreatic duct in the head, the body, and the tail of the pancreas, and evaluating the regularity of the main pancreatic duct and

chronic pancreatitis. ${ }^{1}$ Three studies reported an increased frequency of abnormal pancreatograms in biliary lithiasis, ${ }^{2-4}$ mainly in choledocolithiasis. Choledochal stones would damage the pancreatic duct when passing through the papilla. ${ }^{1}$ Other important factors, however, might have been underestimated. For instance, the effect of aging was not completely evaluated in the studies of Satake $e t a l^{2}$ and Axon $\mathrm{et} \mathrm{al}^{3}$ and alcohol consumption was not estimated in any of the previous studies. On the other hand, two studies reported the absence of a relation between gall stones and pancreatographic changes. ${ }^{56}$ This study including 165 patients with current or past history of biliary lithiasis was carried out to evaluate the actual involvement of gall bladder or choledochal stones in pancreatographic changes, among other factors such as age and alcohol consumption that may also induce pancreatographic changes.
\end{abstract} the presence of stenosis, the regularity or the dilatation of secondary ducts, and the presence of cysts. In addition, we established a score, based on the above parameters, by which pancreatograms were classified as normal or with mild, intermediate, moderate or severe abnormalities. A multivariate analysis (stepwise multiple discriminant analysis) was performed for age, sex, presence of gall stones, presence of choledochal stones. Patients were comparable with controls for sex, alcohol consumption but were younger (55 $v 68$ years, $p<0 \cdot 01$ ). In patients and in controls, the frequency of pancreatographic abnormalities increased significantly with age. The pancreatographic features of patients and controls were not significantly different. In the multivariate analysis, age was the only factor with significant predicting value for pancreatographic abnormalities. In conclusion, biliary lithiasis in itself is not an aetiological factor for chronic pancreatitis, older age being responsible for the abnormalities seen by pancreatography of patients with biliary lithiasis.

(Gut 1995; 36: 761-765)

Keywords: biliary lithiasis, pancreatography.

Biliary lithiasis is a well known cause of acute pancreatitis. Usually biliary lithiasis is not considered as an aetiological factor for chronic pancreatitis. Conversely, based on pancreatographic studies, several authors have suggested that it does increase the risk of developing

\section{Methods}

\section{Patients}

Fifty three controls without biliary lithiasis were selected as controls among 3230 patients who had endoscopic retrograde cholangiopancreatography (ERCP) between 1985 and 1992. There were 31 men and 22 women. The mean (SD) age was $55 \cdot 8(15 \cdot 8)$. All of them had a normal cholangiogram, a normal pancreatogram, and a normal computer tomodensitometry. The indication of ERCP in this control group was epigastric pain in 12 cases $(22.6 \%)$; pain in the right upper quadrant in six $(11 \cdot 3 \%)$, which was associated with cholestasis in six $(11.3 \%)$ cases, or with fever in six $(11.3 \%)$; cholestasis (increase in both serum concentrations of alkaline phosphatases and $\gamma$-glutamyltransferase or total bilirubin) or both in five cases $(9.5 \%)$; investigation for suspected carcinoma of the head of the pancreas in two cases $(3.8 \%)$, pain in the left upper quadrant in two cases $(3.8 \%)$, alcohol misuse in two cases $(3.8 \%)$, and miscellaneous in 12 cases $(22 \cdot 6 \%)$. They did not have either biliary lithiasis or known pancreatic disease (carcinoma of the pancreas, chronic calcifying pancreatitis). The age, the daily consumption of alcohol and tobacco were registered and compared with those of the patients.

During the same period, 260 patients had ERCP for biliary investigation. Sixty nine were excluded: seven had carcinoma of the head of the pancreas, four had papillary carcinoma, six had cholangiocarcinoma, four had papillitis, 12 had chronic calcifying pancreatitis, 36 had 
TABLE I Pancreatographic score (abnormalities considered for scoring were: irregularity of the pancreatic duct, stenosis of the pancreatic duct, irregularity of the side branches, dilatation of the side branches, pancreatic cyst)

\begin{tabular}{lcl}
\hline $\begin{array}{l}\text { Dilatation of the pancreatic duct }(P D) \\
(P D \text { width }(\mathrm{mm}))\end{array}$ & $\begin{array}{l}\text { No of } \\
\text { abnormalities }\end{array}$ & Group \\
\hline Absent & 0 & A: normal pancreatogram \\
(head $\leqslant 4 \cdot 8$; body $\leqslant 3 \cdot 4 ;$ tail $\leqslant 2 \cdot 3)$ & 1 & B: mild changes: grade 1 \\
& 2 & C: intermediate changes: grade 2 \\
& 3 & D: moderate changes: grade 3 \\
Moderate & 0 & E: severe changes: grade 4 \\
$(4 \cdot 8<$ head $\leqslant 6 \cdot 5 ; 3 \cdot 4<$ body $\leqslant 5 ; 2 \cdot 3<$ tail $\leqslant 3)$ & 1 & B: mild changes: grade 1 \\
& 2 & C: intermediate changes: grade 2 \\
Severe & $\geqslant 3$ & D: moderate changes: grade 3 \\
(head $>6 \cdot 5 ;$ body $>5$; tail $>3)$ & 0 & E: severe changes: grade 4 \\
& 1 & D: intermediate changes: grade 2 \\
& $\geqslant 2$ & E: severe changes: grade 4 \\
\hline
\end{tabular}

an attack of acute biliary pancreatitis less than two months before ERCP. Of the remaining 191 patients, 26 had incomplete filling of the pancreatic duct or of its side branches and were excluded. A total of 165 patients were finally selected. Their mean age was $68 \cdot 2$ $(15 \cdot 4)$. There were 84 men $(50.9 \%)$ and 81 women $(49 \cdot 1 \%)$. Sixty four of them $(38 \cdot 7 \%)$ presented with cholangitis, $22(13.3 \%)$ had epigastric pain and cholestasis, $14(8.5 \%)$ had fever and cholestasis without abdominal pain, $12(7 \cdot 3 \%)$ had residual choledochal stones after biliary surgery, $11(6 \cdot 7 \%)$ had jaundice with no fever or abdominal pain, $15(9 \cdot 1 \%)$ had abdominal pain, nine $(5.5 \%)$ had cholestasis. Five groups were defined: group 1: gall bladder stones $(n=35)$, group 2: choledochal stones in patients who had had a cholecystectomy $(n=50)$, group 3: gall bladder and choledochal stones $(n=53)$, group 4: choledochal stones without gall bladder stones $(n=10)$, group 5 : patients who had had a cholecystectomy without choledochal stones. The mean delay between the first symptoms and ERCP was 66.1 days (range 1-1460) and no recent attack of acute pancreatitis occurred during this delay.

ERCP films were examined independently by two observers. A clear delineation of the entire main pancreatic duct up to the tail and of the first and second order side branches was required for assessment. The maximum width of the main pancreatic duct and of its side branches was measured in the head, body, and tail of the pancreas. The regularity and presence of strictures of the main pancreatic duct, the regularity and the dilatation of side branches and their location, the presence of pancreatic cysts, their size, number, and location were recorded. The presence, the number, and size of gall stones or choledochal stones were also registered. The maximal diameter of the common bile duct was measured. All the measurements were made on the $x$ ray films with fine calipers. Measurements were corrected for the magnification factor $(\times 1 \cdot 1)$. The main pancreatic duct was considered appreciably enlarged when its measurement was larger than 6.5 $\mathrm{mm}$ in the head, $5 \mathrm{~mm}$ in the body, and $3 \mathrm{~mm}$ in the tail of the pancreas, in agreement with the international definitions of Cambridge. ${ }^{7}$ The main pancreatic duct was considered as slightly enlarged when the duct width was between 4.8 and $6.5 \mathrm{~mm}$ in the head, 3.4 and
$5 \mathrm{~mm}$ in body, and 2.3 and $3 \mathrm{~mm}$ in the tail of the pancreas. The lower limit was chosen on the basis of previous measurements of the pancreatic duct in the normal population. ${ }^{8-11}$

To describe the exact configuration of the pancreatograms, we established a score, taking into account the width of the main pancreatic duct (head, body, tail), the presence of irregularity or stenosis of the main pancreatic duct, the presence of dilatation or irregularities of the side branches and the presence and the size of pancreatic cysts (Table I). Based on these parameters, pancreatograms were classified as normal (grade 0 ), or with mild (grade 1), intermediate (grade 2), moderate (grade 3 ), or severe (grade 4) abnormalities (Table I).

\section{Data analysis}

Data were entered into the Dbase III+ software. Statistical analysis was performed using the SAS software. The mean differences were tested using Student's $t$ test for the comparison of two groups and by one way analysis of variance for more than two groups. Qualitative variables were compared using the $\chi^{2}$ test with Yates's correction when appropriate and with Fisher's exact test for $2 \times 2$ contingency tables. Linear parametric coefficient correlations were tested to assess the relation existing between age and the common bile duct or main pancreatic duct diameters. Only results with a $p$ value less than 0.01 were considered significant.

Determination of variables that independently influenced the calculated pancreatographic score was done by stepwise discriminant analysis. The variables tested included age, sex, the presence of gall bladder stones, the presence of choledochal stones, the diameter of the common bile duct. The variables were chosen to be included or excluded from the model according to the criteria: (a) the significance value of an $F$ test from an analysis of covariance where the variables already chosen act as covariates and the variable under consideration was the dependent variable; (b) the squared partial correlation for predicting the variable under consideration, controlling for the effects of the variables already selected for the model. Stepdisc procedure from computer SAS software allowed us to perform this stepwise discriminant analysis (SAS Institute, 1988).

\section{Results}

\section{Controls}

Twenty two controls $(41.5 \%)$ had a mean daily consumption of alcohol of $56.5 \mathrm{ml}$. Twenty four of them $(45 \cdot 3 \%)$ smoked and the mean daily consumption of tobacco was $19 \cdot 8 \mathrm{~g}$.

Three patients $(5 \cdot 8 \%)$ had diabetes, three patients $(5 \cdot 8 \%)$ had pancreas divisum, one had incomplete fusion of the dorsal and ventral parts of the pancreas, one ( $2 \%$ ) had a common pancreaticobiliary channel. In one case $(2 \%)$, the papilla was juxtadiverticular. 

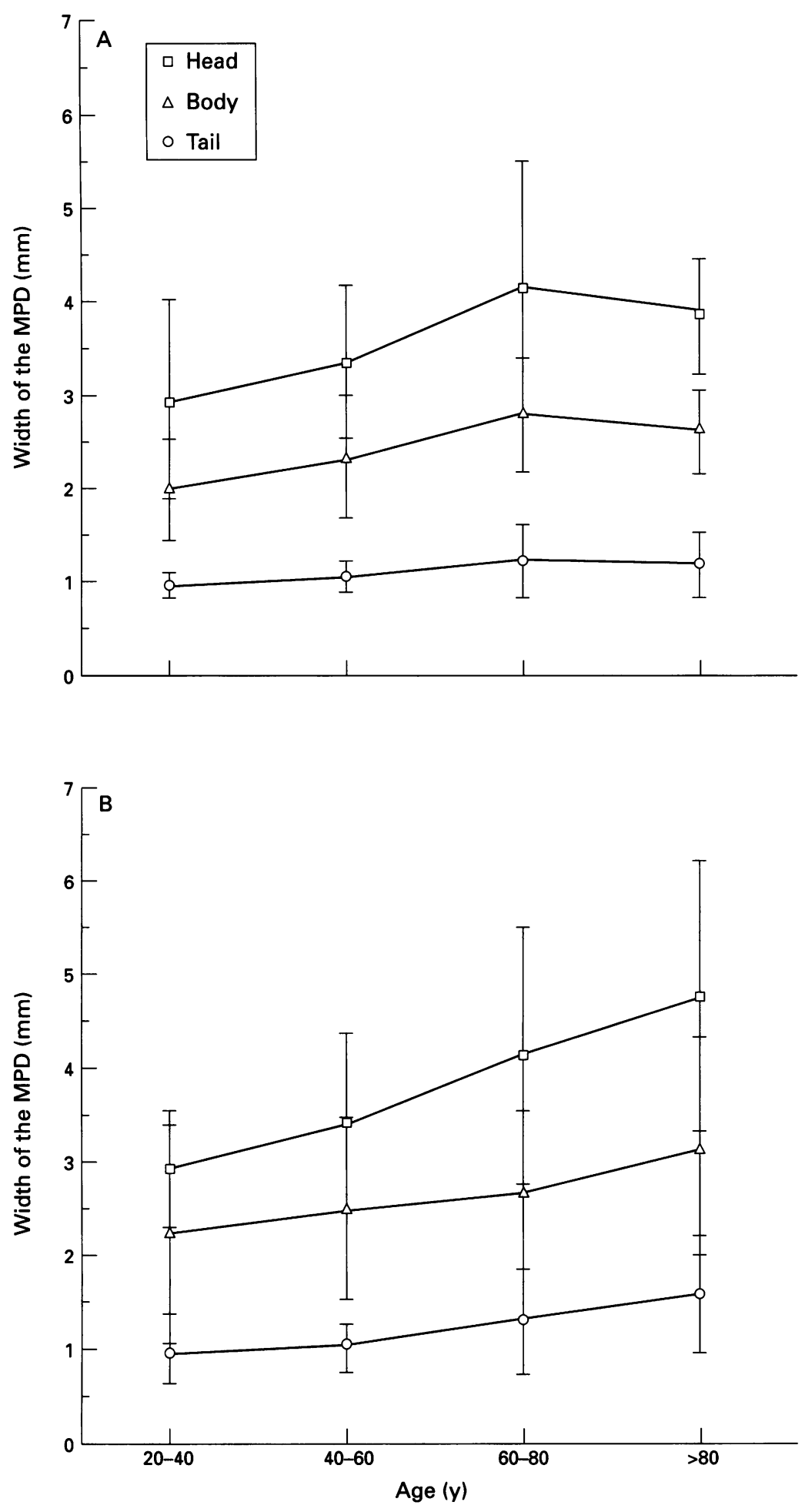

Figure 1: Width of the main pancreatic duct (MPD) in controls (A) and in patients $(B)$, in the head, the body, and the tail of the pancreas (mm, mean (SD)).

Serum concentrations of amylase were normal in 49 patients $(92.5 \%)$. Changes in pancreatographic features were analysed as a function of age (Fig 1A). The pancreatic duct diameter increased with age, in the head, body, and tail of the pancreas. Significant coefficients of correlation between age and main pancreatic duct diameter were found in the head $(r=0.37, \mathrm{p}<0.01)$ body $(r=0.32, \mathrm{p}<0.01)$, and tail of the pancreas $(r=0.30, \mathrm{p}<0.01)$. When considering the pancreatographic score, the frequency of pancreatographic abnormalities significantly increased with age $(p<0.001)$ (Fig 2).
Patients

Fifty three patients $(32 \%)$ had a mean daily consumption of alcohol of $45.5 \mathrm{ml}, 44(26.6 \%)$ smoked and the daily consumption of tobacco was $20.6 \mathrm{~g}$ per day.

Eighty eight patients had gall bladder stones with multiple calculi in 67 cases $(76 \cdot 1 \%)$. The mean size of stones (mm (SD)) was $12.4 \mathrm{~mm}$ (8) when choledochal stones were also present and $11.5 \mathrm{~mm}(6 \cdot 6)$ when there was no choledochal stones.

One hundred and thirteen patients had choledochal stones, a single one was found in 60 cases $(53.9 \%)$. The mean size of the choledochal stones was $11.6 \mathrm{~mm}(7 \cdot 8)$. Fifty patients had had a cholecystectomy with a mean (SD) delay of 3016 days (368).

Nine patients $(5 \cdot 5 \%)$ had diabetes and two $(1 \cdot 2 \%)$ had post viral liver cirrhosis.

Five patients $(3 \%)$ had pancreas divisum and six patients $(3.6 \%)$ had a biliary-pancreatic common channel. In five cases (3\%), the papilla was intradiverticular and in 13 cases $(7 \cdot 9 \%)$ the papilla was juxtadiverticular. Serum concentrations of amylase were normal in 163 cases $(98 \cdot 7 \%)$.

Pancreatograms were also analysed according to age (Fig 1B). The main pancreatic duct diameter increased with age in the head, body, and tail of the pancreas. Significant coefficients of correlation were found between age and the main pancreatic duct diameter in the head $(r=0.38, \mathrm{p}<0.001)$, body $(r=0.30, \mathrm{p}<0.001)$, and tail of the pancreas $(r=0.39, \mathrm{p}<0.001)$. When considering the pancreatographic score, pancreatographic abnormalities significantly increased with age $(p<0.001)$ (Fig 2). The distribution of the pancreatographic abnormalities according to our pancreatographic score clearly showed that moderate and severe pancreatographic (>grade 3) lesions appeared beyond the age of 60 and increased in comparative frequency with age, whereas mild abnormalities (grade 1) decreased (Table II).

Evaluation of the matching between patients and controls

Patients and controls were comparable for sex ratio, proportion of drinkers, and the amount of daily intake of alcohol among drinkers, frequency of abnormal serum concentrations of amylase but not for age and proportion of smokers. Controls were younger than patients $(55.8(15.8) v 68.2(15.4), \mathrm{p}<0.01)$. Main pancreatic duct diameter in the head and body of the pancreas was not significantly different in both groups, but in controls main pancreatic duct diameter was slightly smaller in the tail $(p<0.01)$ (Table III). The frequency of abnormal pancreatograms did not differ among patients and controls (59 of $165(35 \cdot 8 \%) v 22$ of $53(41.6 \%)$ (Table III). The frequency of irregularities of the main pancreatic duct, stenosis of the main pancreatic duct, dilatation of side branches, and presence of pancreatic cysts were not significantly different. Moreover irregular side branches seemed to be more frequent in controls than in patients 10 of $53(18.9 \%) v 14$ of $165(8 \cdot 6 \%)(p=0 \cdot 04)$ (Table III). 


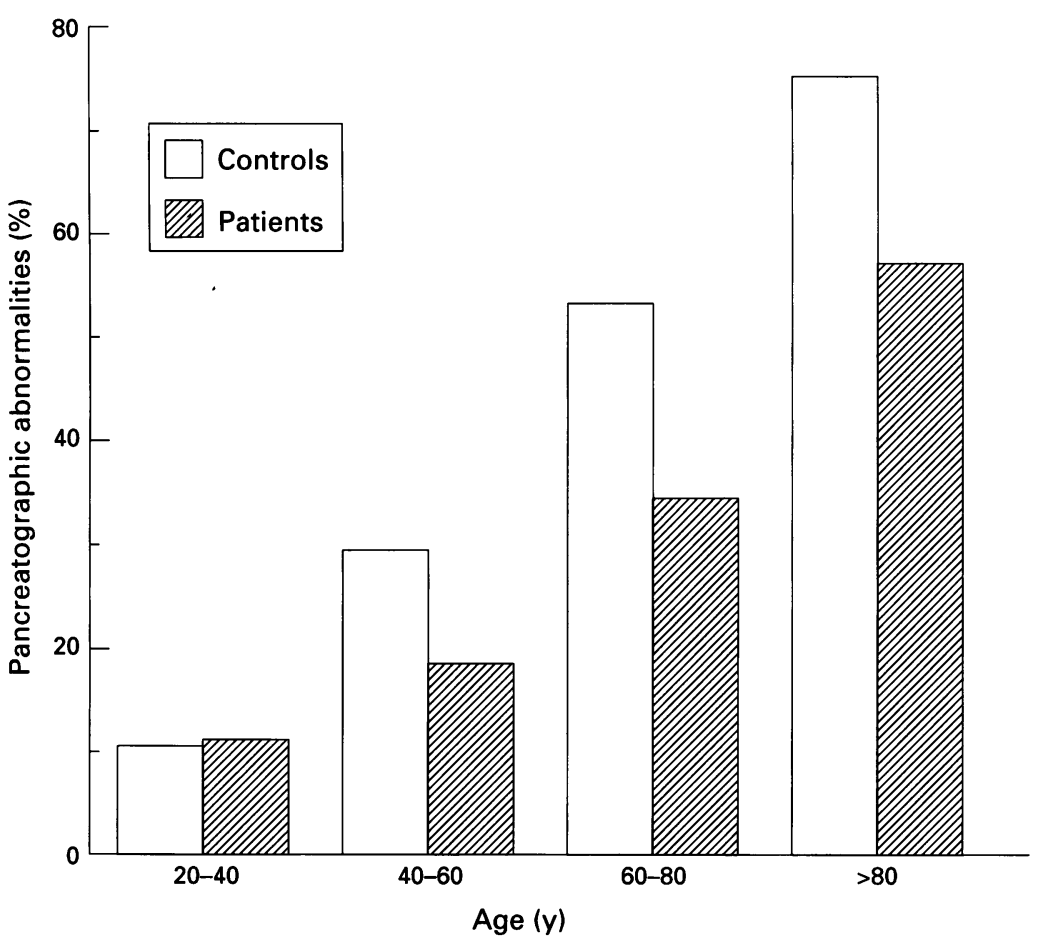

Figure 2: Proportion of abnormal pancreatograms ( $\geqslant$ grade 1) according to age, in controls $\left(\chi^{2} ; p<0.001\right)$ and in patients $\left(\chi^{2} ; p<0.001\right)$.

Stepwise discriminant analysis

Stepwise discriminant analysis was performed for controlling the possible combined effects of aging, sex, gall stones, choledochal stones, width of the biliary duct. In the first step, the variables age, sex, presence of choledochal stones, presence of gall stones, diameter of the biliary duct were entered together in the model. Age was the sole variable to show a significant squared partial correlation $\left(r^{2}=0.01, p=0.0001\right)$. The second, third, and fourth step were successively performed after removal of the variables age, presence of the choledochal stones, width of the common bile duct. At each step, none of the remaining variables showed a significant squared partial correlation. Age was the sole variable with predicting value for the pancreatographic score $\left(r^{2}=0 \cdot 1022, \mathrm{p}=0 \cdot 0001\right)$.

TABLE II Distribution of the severity of pancreatographic abnormalities (score) according to the age of patients

\begin{tabular}{llllc}
\hline $\begin{array}{l}\text { Pancreatographic } \\
\text { score }\end{array}$ & $\begin{array}{l}20-40 \text { years } \\
(n=9)\end{array}$ & $\begin{array}{l}40-60 \text { years } \\
(n=33)\end{array}$ & $\begin{array}{l}60-80 \text { years } \\
(n=81)\end{array}$ & $\begin{array}{l}>80 \text { years } \\
(n=42)\end{array}$ \\
\hline $\begin{array}{l}\text { Grade 1 (mild) (\%) } \\
\text { Grade 2 (intermediate) (\%) }\end{array}$ & 0 & $4(66 \cdot 6)$ & $15(53 \cdot 5)$ & $7(29 \cdot 2)$ \\
Grade 3 (moderate) (\%) & $1(11 \cdot 1)$ & $2(33 \cdot 3)$ & $10(35 \cdot 7)$ & $9(37 \cdot 5)$ \\
Grade 4 (severe) (\%) & 0 & 0 & $2(7 \cdot 1)$ & $6(25)$ \\
Total (\%) & $1(11 \cdot 1)$ & $6(18 \cdot 2)$ & $28(34 \cdot 6)$ & $24(57 \cdot 1)$ \\
\hline
\end{tabular}

TABLE III Comparison of pancreatograms in patients and in controls (S: Student's t test, F: Fisher's exact test)

\begin{tabular}{|c|c|c|c|c|c|c|c|c|c|}
\hline & \multirow{2}{*}{\multicolumn{3}{|c|}{ Width of the MPD in mm (mean, $S D)$}} & \multicolumn{2}{|l|}{$M P D$} & \multicolumn{2}{|c|}{ Side branches } & \multirow{3}{*}{$\begin{array}{l}\text { Pancreatic } \\
\text { cyst } \\
(\%)\end{array}$} & \multirow{3}{*}{$\begin{array}{l}\text { Abnormal } \\
\text { pancreatogram } \\
\text { (\%) }\end{array}$} \\
\hline & & & & \multirow{2}{*}{$\begin{array}{l}\text { Irregularity } \\
(\%)\end{array}$} & \multirow{2}{*}{$\begin{array}{l}\text { Stenosis } \\
(\%)\end{array}$} & \multirow{2}{*}{$\begin{array}{l}\text { Dilatation } \\
(\%)\end{array}$} & \multirow{2}{*}{$\begin{array}{l}\text { Irregularities } \\
(\%)\end{array}$} & & \\
\hline & Head & Body & Tail & & & & & & \\
\hline $\begin{array}{l}\text { Patients }(n=165) \\
\text { Controls }(n=53)\end{array}$ & $\begin{array}{l}4 \cdot 12(1 \cdot 38) \\
3.67(1 \cdot 17) \\
\text { NS (S) }\end{array}$ & $\begin{array}{l}2 \cdot 78(0.99) \\
2 \cdot 48(0.80) \\
\text { NS (S) }\end{array}$ & $\begin{array}{l}1.33(0.57) \\
1 \cdot 10(0 \cdot 29) \\
\mathrm{p}=10^{-2}(\mathrm{~S})\end{array}$ & $\begin{array}{l}6 \cdot 1 \\
3 \cdot 8 \\
\text { NS (F) }\end{array}$ & $\begin{array}{l}2 \cdot 5 \\
0 \\
\text { NS }(F)\end{array}$ & $\begin{array}{l}8 \\
5 \cdot 6 \\
\text { NS (F) }\end{array}$ & $\begin{array}{l}8.6 \\
18.9 \\
p=0.04\end{array}$ & $\begin{array}{l}10 \cdot 3 \\
9.5 \\
\text { NS (F) }\end{array}$ & $\begin{array}{l}35.8 \\
41 \cdot 6 \\
\text { NS (F) }\end{array}$ \\
\hline
\end{tabular}

\section{Discussion}

In this retrospective study, we failed to show any effect of biliary lithiasis on pancreatographic changes. We did not use the Cambridge classification, which was established for chronic pancreatitis because we do not know if the eventual pancreatographic changes associated with biliary lithiasis may be related to chronic pancreatitis. Furthermore, we prefer to describe separately all the parameters of the pancreatograms (Table I). Three previous studies described an increase in pancreatic duct abnormalities caused by biliary lithiasis. $^{2-4}$ Satake et al reported seven of 21 patients with dilatation of the pancreatic duct in gall stone disease (33\%) and 13 of 18 patients with choledochal stones $(72 \%){ }^{2}$ They did not investigate the role of age, or alcohol consumption and they considered only the diameter of the pancreatic duct. Axon et al ${ }^{3}$ studied 53 patients and 12 controls. Pancreatic duct abnormalities were present in $47 \%$ of patients and in $8 \%$ of controls, but the patients with abnormal pancreatic ducts were older than those without pancreatic changes (65 $v 51$ years). The daily intake of alcohol was not given. Misra et al found $47 \%$ of pancreatic duct abnormalities in 53 patients with gall stones disease and in $6 \%$ among 33 controls. ${ }^{4}$ Abnormal pancreatograms were more frequent in patients with choledocolithiasis than in patients who had had a cholecystectomy $(55 \%$ $v 25 \%$ ). Patients and controls were comparable for age (42 $v 38$ years). Neither patients nor controls were classified as drinkers and pancreatograms were categorised according to the Cambridge classification.

In the study of Misra et al age did not influence the frequency of pancreatographic changes among patients except for an increase of the width of the pancreatic duct. Many studies, however, performed at necropsy with or without postmortem pancreatography examination, showed that the frequency of abnormalities of the pancreatic duct and its side branches increased with age. Kreel and Sandin reported dilatation of the pancreatic duct and ductular ectasia mainly located to the head of the pancreas, occurring mostly in older patients. ${ }^{12}$ Nagai and Ohtsubo found pancreatic lithiasis (stones smaller than $3 \mathrm{~mm}$ ) located in side branches of the pancreatic duct, increasing in frequency with age. ${ }^{13}$ Stamm found in 112 unselected necropsy examinations, ductal epithelial changes associated with lipomatosis, fibrosis, and dilatation of the pancreatic duct and its side branches, also correlated with age. ${ }^{14}$ Therefore, histological evidence exists for pancreatic changes increasing in frequency and severity with age. Jones 
et $a l^{6}$ analysing retrograde pancreatography in 101 patients aged 75 or more, found many abnormal pancreatograms: dilated or undulating main pancreatic duct, atrophic or ectasic side branches, filling defects in the main pancreatic duct, cavity formations. Yet, they found no significant relation between the presence of common bile duct stones and pancreatographic changes. Anand et $a l^{15}$ and Neoptolemos et al $^{5}$ showed a linear correlation between the width of the main pancreatic duct and age. The same finding was made in our series in patients with biliary stones and in controls (Figs 1, 2; Table I), confirming an effect of aging on pancreatic duct damage.

The proportion of drinkers and the mean daily consumption of alcohol was comparable in controls and patients. Considering drinkers and non-drinkers, the ratio of abnormal pancreatograms was similar in controls (nine of 22 $v 13$ of $31(40.9 \% v 41 \cdot 2 \%))$ and in patients (19 of $51 v 38$ of $108(37 \cdot 2 \% \quad v 35 \cdot 1 \%)$ ). Alcoholic chronic pancreatitis might have been misdiagnosed in controls and in patients, but the probability of having misdiagnosed alcoholic chronic pancreatitis may be very close in both groups. The controls were younger than the patients $(55.8 v 68.2$ years), but similar pancreatographic changes according to the age were seen in both groups (Figs 1 and 2). Pancreatographic changes do not seem to be related to a recent attack of acute pancreatitis as no acute pancreatitis occurred during the mean delay of 66 days between the onset of symptoms and ERCP; moreover serum concentrations of amylase were comparable among patients and controls.

The diameter of the main pancreatic duct in the tail of the pancreas was smaller in controls $(p=0.01)$, possibly because of the lower age of the controls, as we showed that in controls and in patients the width of the main pancreatic duct was correlated with age (Fig 1). Irregularities of side branches were more frequent in controls than in patients $(18.9 \% v$ $8.6 \%$ ) (Table III). This finding may result from the smaller number of controls $(n=53)$, compared with the number of patients $(n=165)$ or from the higher proportion of smokers among controls, as Bourlière et al ${ }^{16}$ reported the possible effect of smoking on chronic pancreatitis.

In addition, to investigate the role of choledocholithiasis in possible combination with the effects of aging, gall stones, sex or width of the common bile duct, we performed a stepwise discriminant analysis. With that powerful tool, we confirmed that choledocholithiasis and gall stones were not predictive of the presence of pancreatographic abnormalities.
Biliary lithiasis is the most frequent cause of acute pancreatitis, but its participation as an aetiological factor for chronic pancreatitis remained unclear. In this study, based on a careful analysis of pancreatograms, we took into account several factors which, alone or combined with biliary lithiasis, might be associated with pancreatographic changes. If the controls were significantly younger than the patients, they were comparable for alcohol and tobacco consumption. Yet, age correlated well with the frequency and the severity of the pancreatographic changes in both groups. Moreover, in controls, the frequency of abnormal pancreatograms was slightly increased compared with the patients. Finally, multivariate analysis confirmed that age was the sole variable relevant to the pancreatographic score.

We conclude that biliary lithiasis probably does not induce pancreatographic changes and should no longer be considered as a potential aetiological factor of chronic pancreatitis.

1 Misra SP, Dwivedi M. Do gallstones cause chronic pancreatitis? Int f Pancreatol 1991; 10: 97-102.

2 Satake K, Umeyama K, Kobayashi K, Mitani E, Tatsumi S, Yamamoto $S$, et al. An evolution of endoscopic pancreatocholangiography in surgical patients. Surg Gynecol Obstet 1975; 140: 349-54.

3 Axon ATR, Ashton MG, Lintott J. Pancreatograms changes in patients with calculous biliary disease. Br f Surg 1979; in patients with

4 Misra SP, Gulatti P, Choudhary V, Anand BS. Pancreatic duct abnormalities in gallstone disease: an endoscopic retrograde cholangiopancreatography study. Gut 1990; 31 1073-5.

5 Neoptolemos JP, Carr-Locke DL, Kelly KA. Factors affecting the diameters of the common bile duct and pancreatic duct using endoscopic retrograde cholangiopancreatography. Hepatogastroenterology 1991; 38: 243-7.

6 Jones SN, Mc Neil NI, Lees WR. The interpretation of retrograde pancreatography in the elderly. Clin Radiol 1989; 40: 393-6.

7 Axon ATR, Classen M, Cotton PB, Cremer M, Freeny PC Lees WR. Pancreatography in chronic pancreatitis: international definitions. Gut 1984; 25: 1107-12.

8 Kasugai T, Kuno N, Kizu M, Kobayashi S, Hattori K. Endoscopic pancreatocholangiography. II. The pathological endoscopic pancreatocholangiogram. Gastroenterology 1972; 63: 227-34

9 Cotton PB. Cannulation of papilla of Vater by endoscopy and retrograde cholangiopancreatography (ERCP). Gut 1972; 13: 1014-25.

10 Classen M, Hellwig H, Rosch W. Anatomy of pancreatic duct, a duodenoscopic radiological study. Endoscopy 1973; 5: 14-7.

11 Verley PF, Rohrmann CA Jr, Silvis SE, Vennes JA. The normal endoscopic pancreatogram. Radiology 1976; 118 295-300.

12 Kreel L, Sandin B. Changes in pancreatic morphology associated with aging. Gut 1973; 14: 962-70.

13 Nagai H, Ohtsubo K. Pancreatic lithiasis in the aged. Its clinicopathology and pathogenesis. Gastroenterology 1984 86: $331-8$.

14 Stamm BH. Incidence and diagnostic significance of minor pathologic changes in the adult pancreas at autopsy: systematic study of 112 autopsies in patients without known pancreatic disease. Hum Pathol 1984; 15: 677-83.

15 Anand BS, Phil D, Vij JC, Mac HS, Chowdhury V, Kumar A. Effect of aging on the pancreatic ducts: a study based A. Effect of aging on the pancreatic ducts: a study based on endoscopic retrograde

16 Bourliere M, Barthet M, Berthezene P, Durbec JP, Sarles $\mathrm{H}$. Is tobacco a risk factor for chronic pancreatitis and alcoholic cirrhosis? Gut 1991; 32: 1392-5. 